Association for Information Systems

AIS Electronic Library (AISeL)

Wirtschaftsinformatik Proceedings 2005

Wirtschaftsinformatik

February 2005

\title{
Sourcing von Finanzprozessen - Ein Modell zur simultanen Bewertung von Economies of Scale und Scope
}

\author{
Wolfgang König \\ Universität Frankfurt am Main \\ Daniel Beimborn \\ Universität Frankfurt am Main \\ Jochen Franke \\ Universität Frankfurt am Main \\ Tim Weitzel \\ Universität Frankfurt am Main
}

Follow this and additional works at: http://aisel.aisnet.org/wi2005

\section{Recommended Citation}

König, Wolfgang; Beimborn, Daniel; Franke, Jochen; and Weitzel, Tim, "Sourcing von Finanzprozessen - Ein Modell zur simultanen Bewertung von Economies of Scale und Scope" (2005). Wirtschaftsinformatik Proceedings 2005. 89.

http://aisel.aisnet.org/wi2005/89

This material is brought to you by the Wirtschaftsinformatik at AIS Electronic Library (AISeL). It has been accepted for inclusion in Wirtschaftsinformatik Proceedings 2005 by an authorized administrator of AIS Electronic Library (AISeL). For more information, please contact elibrary@aisnet.org. 
In: Ferstl, Otto K, u.a. (Hg) 2005. Wirtschaftsinformatik 2005: eEconomy, eGovernment, eSociety; 7. Internationale Tagung Wirtschaftsinformatik 2005. Heidelberg: Physica-Verlag

ISBN: 3-7908-1574-8

(C) Physica-Verlag Heidelberg 2005 


\title{
Sourcing von Finanzprozessen - Ein Modell zur simultanen Bewertung von Economies of Scale und Scope
}

\author{
Wolfgang König, \\ Daniel Beimborn, Jochen Franke, Tim Weitzel \\ Universität Frankfurt am Main
}

\begin{abstract}
Zusammenfassung: Während viele Unternehmen Fortschritte in der IuKTechnologie zur Automatisierung ihrer primären Prozesse, zu einer internen und externen Vernetzung und letztlich zur Neuaufteilung und Optimierung der gesamten Wertschöpfungskette genutzt haben, wurden Sekundärprozesse selten als eigener Optimierungsgegenstand betrachtet. In einer empirischen Studie zu sekundären Finanzprozessen in den 1.000 größten deutschen Unternehmen (ohne Banken und Versicherungen) wird gezeigt, dass vor einer Outsourcing-Entscheidung kulturelle Hürden der Entscheider zu überwinden sind. Es zeigt sich insbesondere, dass die individuelle Outsourcing-Erfahrung der entscheidenden Manager von Bedeutung ist und dass Economies of Scale und Scope getrennt betrachtet und damit mögliche Trade-offs häufig ignoriert werden. Zur Erweiterung der reinen Outsourcing-Entscheidung im Hinblick auf viel versprechende Modelle des kooperativen Outsourcing wird untersucht, wer geeignete Partner für eine selektive Auslagerung von Teilprozessen durch eine Kreuzung der eigenen Wertschöpfungskette mit derjenigen des Partners (Value Chain Crossing) sein können. Aufbauend auf diesen empirischen Ergebnissen wird ein Simulationsmodell vorgestellt, welches insbesondere die simultane Betrachtung verschiedener Einflussfaktoren der Outsourcing-Entscheidung erlaubt.
\end{abstract}

Schlüsselworte: Financial Chain Management, Finanzprozesse in Nicht-Banken, Outsourcing

\section{Einleitung}

Während in vielen Unternehmen Fortschritte in der IuK-Technologie zur Automatisierung der primären Prozesse, zu einer internen und externen Vernetzung und letztlich zur Neuaufteilung und Optimierung der gesamten Wertschöpfungskette genutzt wurden, zwingt der Wettbewerb zur ständigen Suche nach neuen Effizienzquellen. In diesem Zusammenhang wird insbesondere Outsourcing als Mittel 
zur Konzentration auf Kernkompetenzen und zur Neugestaltung der Wertschöpfungskette diskutiert.

Basierend auf einer empirischen Studie mit den 1.000 größten deutschen Unternehmen (ohne Banken und Versicherungen) wird in diesem Beitrag gezeigt,

- dass vor einer Outsourcing-Entscheidung kulturelle Hürden durch die Entscheider zu überwinden sind, die sich vor allem in der Einschätzung der Prozesskompetenz der Dienstleister niederschlagen,

- inwieweit Economies of Scale und Scope bedeutsam für das Outsourcing von Teilprozessen sind und wie diese zusammenhängen und

- wer geeignete Partner für ein selektives Outsourcing von Teilprozessen durch eine Kreuzung der eigenen Wertschöpfungskette mit der Wertschöpfungskette des Partners (Value Chain Crossing) sein können.

Vor diesem Hintergrund wird ein formales Outsourcing-Modell entwickelt, das durch die simultane Berücksichtigung von Economies of Scale und Scope optimale (Teil-)Prozessbündelungskonstellationen ermitteln kann.

Als Untersuchungsgegenstand wurden Finanzprozesse in Großunternehmen gewählt, da diese zum einen als typische Sekundärprozesse noch deutliches Optimierungspotenzial vermuten lassen. Zum anderen bieten Finanzprozesse durch ihre prinzipiell durchgängige Digitalisierbarkeit einen idealen Anwendungsfall wirtschaftsinformatischer Methoden zur Prozessoptimierung. Im Gegensatz zu weiten Teilen der Outsourcing-Literatur, die sich vorwiegend mit der Analyse singulärer Erfolgsfaktoren von Outsourcing befasst, wird auf dieser Grundlage ein SourcingSimulationsmodell entwickelt, das die Wirkungen verschiedener Einflussfaktoren wie Economies of Scale und Scope simultan analysiert.

Der nächste Abschnitt gibt einen Überblick über die Literatur zu Outsourcing und stellt insbesondere den Übergang von IT-Outsourcing hin zum Auslagern von Geschäftsprozessen dar. Danach wird eine generische Financial Chain als Grundlage der weiteren Untersuchungen eingeführt (Abschnitt 3.1) und bezüglich Economies of Scale und Scope (Abschnitt 3.2) sowie Chancen und Herausforderungen für ein Outsourcing untersucht (Abschnitt 3.3). Zur simultanen Bewertung des Einflusses dieser Argumente wird in Abschnitt 4.1 die Grundlage für ein formales Simulationsmodell gelegt, das Outsourcing-Entscheidungen verschiedener Akteure einer Branche oder eines Marktsegmentes nachbildet und daraus resultierende strukturelle Effekte aufzeigt. Eine hiermit adressierbare Frage ist: In welchen Situationen ist kooperatives Sourcing (Cooperative Sourcing) und das Zusammenlegen einzelner Geschäftsprozesse eine vorteilhafte Alternative zu M\&A-Aktivitäten und damit dem Fusionieren ganzer Unternehmen? Ein mögliches Beispielszenario wird in Abschnitt 4.2 diskutiert, Abschnitt 4.3 zeigt in einem Ausblick die zukünftige Weiterentwicklung des Modells auf. 


\section{Outsourcing}

Der externe Bezug von IT-Dienstleistungen ist kein neues Phänomen. Bereits in den 60er Jahren übernahmen Systemhäuser, „service bureaus“ und professionelle Systemmanagement-Anbieter IT-Dienstleistungen und boten diese anderen Unternehmen an. IT-Hardware war relativ teuer und externe Dienstleister stellten Timesharing oder Processing Services zur Verfügung [CuWe03]. Im Jahre 1963 war EDS das erste Unternehmen, das die gesamten Datenverwaltungsdienste eines anderen Unternehmens übernahm [DiGo ${ }^{+}$04; LaHi93].

Der steigende Bedarf an IT-Applikationen und Dienstleistungen sowie das gleichzeitig knappe Angebot an qualifiziertem IT-Personal führte in den 70er Jahren da$\mathrm{zu}$, dass Manager benötigte Anwendungen extern erstellen und pflegen ließen.

Die rasante Evolution der Informationstechnologie und das Aufkommen von günstigen PCs traf die IT-Dienstleister zu Beginn der 80er Jahre schwer $\left[\mathrm{LeHu}^{+} 03\right]$. Der vormalig starke Outsourcing-Trend im IT-Bereich wurde unterbrochen und Unternehmen tendierten dazu, vermehrt eigene IT-Abteilungen zu kultivieren. Vertikale Integration und die Kontrolle der gesamten Wertschöpfungskette standen im Fokus. Viele Unternehmen bauten die eigene IT-Abteilung aus, welche die verfügbare Informationstechnologie zu einem spezifisch auf das Unternehmen angepassten System entwickelte. Die externe und interne Integration der Informationssysteme wurde zur vordringlichen Herausforderung [ $\mathrm{LeHu}^{+} 03$ ].

Mit dem in der Literatur vielfach zitierten Kodak-Deal im Jahr 1989 wurde ein neuer Outsourcing-Trend angestoßen [KeWi96; LoVe95]. Der Betrieb von zuvor selbst erstellter IT-Infrastruktur wurde an externe Dienstleister im Rahmen eines IT-Outsourcing (ITO) abgegeben. Bei Earl wird ITO dabei als die Auslagerung Hardware-orientierter IT-Aktivitäten verstanden [Ear196].

Die Entwicklung leistungsfähiger Netzwerke und insbesondere des Internets führten zum Konzept des Application Service Provisioning (ASP) [Tebb03]. ASP umfasst den Betrieb von Applikationen im Rechenzentrum eines Dienstleisters mit der Bereitstellung der Software über ein Netzwerk basierend auf einem selektiven Outsourcing einzelner Applikationen [DeCu03; $\mathrm{KeKr}^{+} 02$ ]. Dabei lässt sich ASP von ITO in der Form abgrenzen, dass ASP standardisierte Lösungen ohne aufwändige Anpassungen an die Kundenerfordernisse bereitstellt (häufig gängige Desktop-Applikationen oder branchenspezifische Standardsoftware), während ITO umfassender auch stark auf einzelne Kunden zugeschnittene Lösungen anbietet und sich neben den Applikationen auch um den Betrieb der zugrunde liegenden Technologien kümmert [Ried03].

Die Auslagerung gesamter Geschäftsprozesse oder einzelner Module mit der zugrunde liegenden IT im Rahmen eines Business Process Outsourcing (BPO) stellt den jüngsten Trend dar [RoCo04; Weer $\left.{ }^{+} 03\right]$. BPO wird dabei als die Auslagerung einzelner spezifischer Geschäftsprozesse verstanden [HaMe00]. Im Ge- 
gensatz zu klassischem ITO wird hier die Informationstechnologie nicht quer über mehrere Geschäftsprozesse oder Unternehmensfunktionen hinweg ausgelagert. Vielmehr wird orthogonal hierzu ein einzelner Prozess inklusive der zugrunde liegenden IT einem externen Provider übergeben [HaMe00].

BPO stellt zurzeit das am schnellsten wachsende Segment im Outsourcing-Markt dar [RoCo04]. Für die Umsätze im ITO-Bereich wird in Großbritanien und Europa ein Wachstum von 154 Mrd. EUR im Jahr 2002 auf über 200 Mrd. EUR im Jahr 2005 prognostiziert [Will ${ }^{+} 04$ ]. Das BPO-Segment ist dagegen allein im Jahr 2002 um $25 \%$ gewachsen, insgesamt wird für 2005 ein Umsatz von $72 \mathrm{Mrd}$. EUR in diesem Segment prognostiziert [Will ${ }^{+} 04$ ]. Gleichzeitig sind die Spezifika dieses Typs von Outsourcing in der wissenschaftlichen Literatur bislang kaum adressiert [Ried03; RoCo04].

Während das Outsourcing von IT mittlerweile zu einem integralen Bestandteil des IT-Managements geworden ist [Rock $\left.{ }^{+} 96\right]$, lassen sich weiter gefasste Analysen, welche die verschiedenen Formen des Outsourcing (wie beispielsweise auch ein kooperatives Sourcing (Zusammenlegung) einzelner Prozessteile von Wettbewerbern) in den Entscheidungsprozess einbeziehen, nicht finden.

Die in neueren Arbeiten zum Resource Based View zu findende Erkenntnis, dass der Geschäftsprozess im Fokus der Betrachtung und Analyse des Wertbeitrages der IT stehen sollte, führt zu einer stärker prozess- oder ressourcenorientierten Sichtweise des Unternehmens [Baru ${ }^{+}$95; Melv $^{+}$04]. IT selbst wird oft als ,enabling factor" in einem prozessorientierten Unternehmen gesehen [FeWi98]. Insbesondere in Unternehmen, die bereits in einem hohen Maße prozessorientiert arbeiten, wird die IT weniger als isolierte Funktion, sondern vielmehr als integraler Bestandteil einzelner Prozesse betrachtet, besonders auch über verschiedene Unternehmensfunktionen hinweg [BrRo03]. Daneben deuten die Konzentration auf Kernkompetenzen und der Zukauf aller peripheren Aktivitäten auf einen Wandel in der Art und Weise hin, wie Organisationen gestaltet und geleitet werden [FeWi98; PrHa90]. Die Wahl des Umfanges auszulagernder IT wird dementsprechend in Zukunft weniger funktionsorientiert auf Ebene der IT, sondern prozessorientiert auf Ebene des Geschäftsprozesses entschieden. Dementsprechend ist die Frage nach dem optimalen Sourcing-Mix des Unternehmens zu stellen: Welche Ressourcen sollen intern erstellt, welche sollen von welchem Anbieter extern bezogen werden? Die optimale Kombination und Orchestrierung von externen Ressourcenbündelungskonzepten wie ITO, ASP und BPO sowie internen Ansätzen wie der Gründung einer Shared Service Organization stellt dabei eine komplexe, strategische Entscheidung dar.

Auf der Ebene von ITO wurden hier beispielsweise Fragen des selektiven oder totalen Outsourcing von IT-Funktionen [Laci ${ }^{+}$96] und der Auswahl einzelner ITFunktionen diskutiert [RoCo03]. Die Wahl der geeigneten Schnitte durch die Teilprozesse und die Bündelung einzelner Ressourcen sind bislang nur unzureichend betrachtet worden. Nach einer Identifikation der Schwachstellen in Ge- 
schäftsprozessen ist ein ganzheitliches Sourcing-Modell zur Bestimmung der optimalen Sourcing-Konfiguration von zentraler Bedeutung. Teilfragen des Modells (etwa in Bezug auf selektives und totales ITO) sind bereits in der Literatur adressiert. Ein Modell, das eine Beurteilung der Gesamtsituation des Unternehmens erlaubt, existiert bislang nicht.

\section{Financial Chain Management und Outsourcing}

Im Folgenden wird zunächst das Konzept der Financial Chain vorgestellt sowie in die durchgeführte empirische Studie eingeführt. Es werden in einem ausgewählten Prozess, der Financial Chain, mögliche Sourcing-Optionen und Sourcing-Partner evaluiert sowie die Rolle von Economies of Scale und Economies of Scope diskutiert.

\subsection{Financial Chain}

Für viele Primärprozesse wurden Fortschritte in der IuK-Technologie zur Automatisierung, zur internen und externen Vernetzung und letztlich zur Neuaufteilung der Wertschöpfungskette genutzt. Dabei lassen sich Primär- oder Kernprozesse als diejenigen Prozesse definieren, die einen direkten Beitrag zur Wertschöpfung des Unternehmens leisten, wohingegen Sekundärprozesse Unterstützungs- und Managementprozesse umfassen [Hein02].

Das Management der Finanzflüsse (als typischer Sekundärprozess in NichtBanken) ist bislang in den wenigsten Fällen durch Prozessoptimierungsmaßnahmen erfasst worden und immer noch stark durch die Aufbauorganisation bestimmt. Wird in Betracht gezogen, dass die Finanzprozesse im Durchschnitt ein Viertel der gesamten IT-Budgets benötigen, lohnt ein Blick auf potenzielle Verbesserungsmöglichkeiten in diesem Bereich [Skie ${ }^{+}$04].

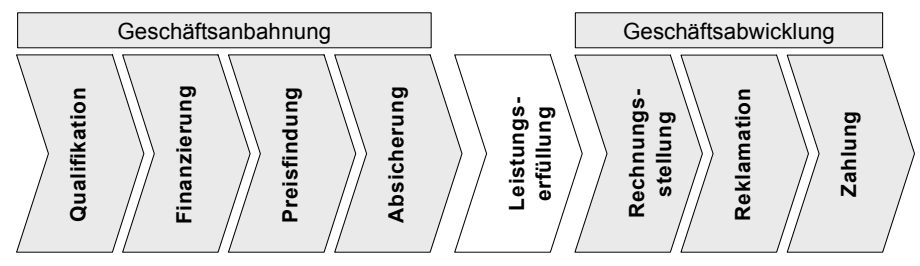

Abbildung 1: Generische Financial Chain [Pfaf ${ }^{+} 03$ ]

Die Aktivitäten in einer die physischen Leistungsprozesse der Supply Chain begleitenden Financial Chain fallen in den meisten Unternehmen unter die unterstützenden Sekundärprozesse, die im Gegensatz zu den primären Leistungserstel- 
lungsprozessen keinen direkten Beitrag zur Wertschöpfung der Unternehmen liefern. Sie liegen dadurch viel zu selten im Blickpunkt des Top-Managements [Skie ${ }^{+}$04]. Die aus sieben Teilschritten bestehende Financial Chain (Abbildung 1) ist immanent mit den Prozessen der physischen Leistungserstellung in der Supply Chain verknüpft und lässt sich in die durch die physische Leistungserfüllung getrennten Bereiche Geschäftsanbahnung und Geschäftsabwicklung einteilen [Pfaf $\left.{ }^{\dagger} 03\right]$. Die Geschäftsanbahnung beginnt mit der Qualifikation der Geschäftspartner, indem Identität und Bonität des Kunden überprüft werden. Danach wird die Finanzierung des Geschäftes geregelt, etwa durch einen Lieferantenkredit oder Leasing. Es folgen Preisverhandlung und -festsetzung (Preisfindung) und anschließend die Angebotserstellung sowie die Absicherung von Währungs-, Transport- und Kreditausfallrisiken. Die Erfüllung des Geschäftes erfolgt in den Prozessen der physischen Supply Chain, die im Rahmen des Supply Chain Managements mit Lieferanten und Kunden synchronisiert werden. Nach dem Erfüllungsgeschäft beginnt die Geschäftsabwicklung mit der Rechnungsstellung und deren Versand sowie eventuellen Rechnungsreklamationen durch den Kunden. Sind Beanstandungen der Rechnung beseitigt, erfolgt die Zahlung der Rechnung.

In einer Studie zur Financial Chain wurde ein Fragebogen mit 35 teils weiter detaillierten, offenen und geschlossenen Fragen auf acht Seiten an die Finanzverantwortlichen der umsatzstärksten 1.000 Unternehmen (ohne Finanzdienstleister) in Deutschland gesendet [Skie ${ }^{+}$04]. Der Fragebogen gliederte sich in detaillierte Fragestellungen zu den sieben Teilprozessen sowie in übergreifende Fragen zur gesamten Financial Chain. Im Vorlauf der Studie wurde der Fragebogen in PreTests und Expertengesprächen auf Verständlichkeit und inhaltliche Konsistenz geprüft und angepasst. Weiterhin wurden alle Unternehmen telefonisch kontaktiert, um die Chief Financial Officers (CFOs) zu identifizieren. Diesen wurde anschließend der Fragebogen direkt zugeschickt. Die Studie war dabei auf die Branchen ausgerichtet, in denen die Prozesse der Financial Chain keine Primär- oder Kernprozesse darstellen, d. h. Banken und sonstige Finanzdienstleister wurden ausgeschlossen. In einer schriftlichen und einer telefonischen Nachfassaktion wurden alle Finanzverantwortlichen erneut kontaktiert und zur Mitarbeit aufgefordert oder nach Gründen für die bislang ausbleibende Beantwortung des Fragebogens befragt. Die Rücklaufquote der Befragung lag bei 103 verwendbaren Datensätzen (10,3\%). Ausgewählte Ergebnisse dieser Studie finden sich im nächsten Abschnitt.

\subsection{Outsourcing der Financial Chain - Eine Betrachtung der Economies of Scale und Economies of Scope}

Die Financial Chain wird als Sekundärprozess zur Unterstützung der Kerntätigkeit [Port85] nicht mit der Absicht gestaltet und optimiert, daraus einen strategischen Wettbewerbsvorteil in Form eines Alleinstellungsmerkmals zu erhalten. Entsprechend bietet sich die Überlegung an, diese Teilprozesse an einen entsprechenden 
Dienstleister auszulagern. Im Folgenden sollen die Einschätzungen der Auslagerungsfähigkeit eines Teilprozesses vor dem Hintergrund der in der Literatur fokussierten Outsourcing-Determinanten „Economies of Scale“ bzw. „Economies of Scope" dargelegt werden.

Als wesentlicher Vorteil des Outsourcing werden i. d. R. Einsparungen aus Skaleneffekten genannt, die sich durch Bündelung gleicher Prozesse mehrerer Organisationseinheiten oder Unternehmen beim Insourcer ergeben [CaHa02; $\mathrm{MaMe} 02$; Scho97]. Dem gegenüber steht das Argument, dass große Unternehmen diese erreichbaren Economies of Scale schon innerhalb ihrer eigenen Organisation realisieren können, dass also Skalenerträge sowohl generell erschöpfbar sind als auch im eigenen Unternehmen bereits ausgereizt werden [Ear196; Laci $\left.{ }^{+} 96\right]$. Einige Teilprozesse der Financial Chain sind - abhängig von der Branche - in solchem $\mathrm{Maß}$ repetitiv, dass - unter Vernachlässigung von temporären Schwankungen des Prozessvolumens - die echten Fixkosten eine untergeordnete Rolle spielen. Nimmt man eine lineare Kostenfunktion an, wäre das Prozessvolumen so hoch, dass die Durchschnittskosten hinreichend an die Grenzkosten angenähert sind. So sehen 63,8\% der antwortenden Unternehmen keine oder nur für die Minderheit der Teilprozesse Skalenerträge, die durch Outsourcing zusätzlich realisiert werden könnten (Tabelle 1: 1.1). Nur 17,6\% sind anderer Ansicht und halten für große Teile der Financial Chain auch bei großen Unternehmen Einsparungen durch Skaleneffekte noch für möglich.

Das Ausbleiben von zusätzlichen Skalenerträgen durch Outsourcing hängt jedoch nicht nur davon ab, in welchem Umfang die jeweiligen Teilprozesse schon im eigenen Unternehmen ausgeführt werden, sondern auch von der Prozessheterogenität. Zusätzliche Skaleneffekte können nur realisiert werden, wenn in den Unternehmen einer Branche diese Teilprozesse hinreichend standardisiert ablaufen oder entsprechend homogenisiert werden können. Hier sind die Befragten allerdings recht skeptisch. Nicht einmal die Hälfte (42,9\%) der befragten Unternehmen hält eine branchenweite Prozessstandardisierung für möglich (Tabelle 1: 1.2). Diese Einschätzung muss bei einer weiteren Analyse des Verhältnisses interner und externer Skaleneffekte berücksichtigt werden.

Ein mit den Economies of Scale verbundenes Argument für Outsourcing ist die Flexibilisierung der operativen Kosten [MaMe02]. Dazu wird der jeweilige Teilprozess ausgelagert (BPO), als Dienstleistung bezogen und auf Basis der Durchsatzzahlen abgerechnet. So kann das Unternehmen näherungsweise vollständig Fixkosten abbauen und maximale Kostenreduktionen realisieren, wenn die Durchsatzzahlen sinken. Diese Flexibilisierung ist jedoch auf Seiten des Insourcers insbesondere dann möglich, wenn er Skaleneffekte realisieren kann; ansonsten bleibt er genauso auf den Fixkosten sitzen wie vorher das outsourcende Unternehmen. Vorteile aus der Flexibilisierung können dann nur noch realisiert werden, wenn diese Durchsatzzahlen der einzelnen Kunden nicht zu stark positiv korreliert sind. Es ergibt sich ein geteiltes Bild, knapp die Hälfte der Unternehmen ist der An- 
sicht, ein Insourcer könne keine zusätzlichen Skaleneffekte realisieren (Tabelle 1: $1.3)$.

\begin{tabular}{|c|c|c|c|c|c|c|}
\hline $\begin{array}{l}\text { Manager beurteilen Aussa- } \\
\text { gen zu Economies of Scale }\end{array}$ & 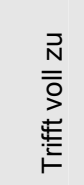 & 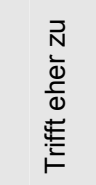 & 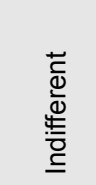 & 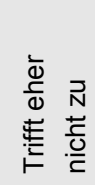 & 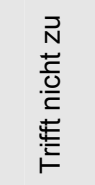 & $\begin{array}{l}\frac{\vec{c}}{0} \\
\frac{0}{\bar{c}} \\
\frac{\varrho}{\bar{N}} \\
3\end{array}$ \\
\hline $\begin{array}{l}\text { 1.1 Auslagerung von Teilpro- } \\
\text { zessen vorteilhaft, da Ska- } \\
\text { leneffekte beim Insourcer } \\
\text { realisiert werden können } \\
(\mathrm{n}=91) \text {. }\end{array}$ & $1,1 \%$ & $15,4 \%$ & $19,8 \%$ & $34,1 \%$ & $29,7 \%$ & $0,0 \%$ \\
\hline $\begin{array}{l}\text { 1.2 Branchenweit standardi- } \\
\text { sierte Prozesse denkbar } \\
(n=98) \text {. }\end{array}$ & $9,2 \%$ & $33,7 \%$ & $12,2 \%$ & $23,5 \%$ & $19,4 \%$ & $2,0 \%$ \\
\hline $\begin{array}{l}\text { 1.3 Prozesse weisen schon im } \\
\text { eigenen Unternehmen ein } \\
\text { derart hohes Transakti- } \\
\text { onsvolumen auf, dass } \\
\text { Outsourcing keine Kos- } \\
\text { teneinsparungen durch } \\
\text { zusätzliche Skaleneffekte } \\
\text { mehr realisieren kann } \\
(n=92) \text {. }\end{array}$ & $7,6 \%$ & $38,0 \%$ & $21,7 \%$ & $20,7 \%$ & $6,5 \%$ & $5,5 \%$ \\
\hline
\end{tabular}

Tabelle 1: Economies of Scale in der Financial Chain

Im Gegensatz zu Skaleneffekten beschreiben Economies of Scope Verbundeffekte aus der gemeinsamen Fertigung von Produkten [PaWi81] oder auch der gemeinsamen Bearbeitung verschiedener Prozessschritte. So sind z. B. dann Economies of Scope zu beobachten, wenn es effizient ist, für unterschiedliche Prozessstufen die gleichen Ressourcen, etwa den gleichen Sachbearbeiter, einzubringen [Teec80]. Auch werden erst durch an verschiedenen Stellen vorhandene Kundendaten in einer gemeinsamen Sicht Kundenprofilanalysen möglich.

Sind Teilprozesse aufgrund ihrer grundsätzlichen Eigenschaften und Anforderungen stark miteinander verzahnt, so lassen sich häufig Vorteile aus Economies of Scope realisieren. Bei einer Outsourcing-Entscheidung ist dann ein Trade-off zwischen möglichen Economies of Scale und bestehenden Economies of Scope zu berücksichtigen. So sollten beispielsweise bei einer Dominanz von Economies of Scope die jeweiligen Teilprozesse nicht oder aber nur zum gleichen OutsourcingDienstleister outgesourct werden. Das uneingeschränkte Isolieren von Teilprozessen ist dann oftmals gar nicht oder nur zu hohen Koordinationskosten möglich [Bruc98]. 


\begin{tabular}{|c|c|c|c|c|c|c|}
\hline $\begin{array}{l}\text { Manager beurteilen Aussa- } \\
\text { gen zu Economies of Scope }\end{array}$ & 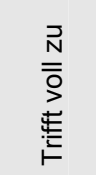 & 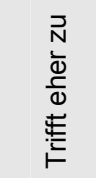 & 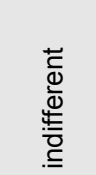 & 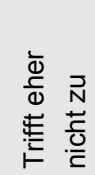 & 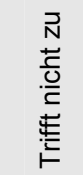 & $\begin{array}{l}\frac{t}{\mathrm{U}} \\
\frac{\mathrm{O}}{\mathrm{E}} \\
\frac{\mathrm{m}}{\mathrm{d}} \\
3\end{array}$ \\
\hline $\begin{array}{l}\text { 2.1 Die Teilprozesse der FC } \\
\text { sind so eng miteinander } \\
\text { verzahnt, dass ein selekti- } \\
\text { ves Outsourcing an jeweils } \\
\text { spezialisierte Dienstleister } \\
\text { nicht effizient sein kann } \\
(n=92) \text {. }\end{array}$ & $17,4 \%$ & $52,2 \%$ & $13,0 \%$ & $9,8 \%$ & $6,5 \%$ & $1,1 \%$ \\
\hline $\begin{array}{l}2.2 \text { In der Integration der Teil- } \\
\text { prozesse liegt ein höheres } \\
\text { Optimierungspotenzial als } \\
\text { in der getrennten Optimie- } \\
\text { rung der Teilprozesse } \\
(n=92) .\end{array}$ & $22,8 \%$ & $54,3 \%$ & $15,2 \%$ & $1,1 \%$ & $3,3 \%$ & $3,3 \%$ \\
\hline
\end{tabular}

Tabelle 2: Economies of Scope in der Financial Chain

Mit 69,6\% sieht eine Zweidrittel-Mehrheit der Unternehmen in den Prozessen der Financial Chain eine starke Verzahnung als gegeben, sodass selektives Outsourcing der Teilprozesse der Financial Chain problematisch wäre (Tabelle 2: 2.1). Dies wird auch durch die Antworten auf die Frage gestützt, ob die getrennte Optimierung der Teilprozesse mehr Einsparpotenzial verspricht als deren Integration (Tabelle 2: 2.2). So geben 77,1\% der befragten Unternehmen an, dass die Integration, d. h. die Abstimmung und die Optimierung der Schnittstellen zwischen den einzelnen Teilprozessen, ein höheres Einsparpotenzial bietet.

Die Betrachtung der Interdependenzen zwischen Economies of Scale und Economies of Scope zeigt, dass Unternehmen i. d. R. schwerpunktmäßig einen der beiden Effekte sehen oder berücksichtigen. So gaben die Antwortenden, die zusätzliche Skaleneffekte beim Insourcer für realisierbar sahen, deutlich häufiger an, keine Economies of Scope zwischen den Teilprozessen sehen zu können und deswegen selektives Outsourcing für sinnvoll zu halten und umgekehrt (hoch signifikante Korrelation nach Pearson: $\mathrm{r}=0,51, \mathrm{p} \leq 0,01$ ).

Zusammenfassend konnte ein von Managern unzureichend wahrgenommener Trade-off zwischen Economies of Scale und Economies of Scope beobachtet werden. Daher wird im Folgenden nach der Untersuchung des Status quo zum Outsourcing der Financial Chain ein Modell zur simultanen Berücksichtigung beider Determinanten entwickelt. 


\subsection{Outsourcing der Financial Chain}

Das Outsourcing von Teilen der Financial Chain (BPO) zu einem spezialisierten Insourcer durch Kreuzung der eigenen Wertschöpfungskette mit der des Insourcers stellt eine Option dar, ausgezeichnete Prozessleistungen auch für einen ansonsten nur unzureichend optimierten Sekundärprozess erreichen zu können. Aus Sicht des Insourcers stellt der Prozess dann einen Kernprozess dar, da dieser dem Outsourcer als eigene Dienstleistung angeboten wird und einen unmittelbaren Bezug zur Wertschöpfung des Insourcers hat.

Neben Economies of Scale stellt die potenziell höhere Kompetenz des Insourcers oder die mangelnde Kompetenz inhouse einen Grund für ein Outsourcing dar [DiHe01]. Da Outsourcing die Wertschöpfungskette eines Unternehmens durch Spezialisierung nachhaltig ändern kann, spielt insbesondere die Einschätzung der Kompetenz der Dienstleister im Vergleich zur eigenen Kompetenz eine tragende Rolle. Die aus der Automobilindustrie bekannte Weisheit „don't raise the cattle for your leather seats“ beschreibt dabei bildhaft den Effekt, durch eine Fokussierung auf die eigenen Kernkompetenzen und die Reduzierung der vertikalen Integration einen nachhaltigen Beitrag zum Unternehmenswert zu leisten. Im klaren Gegensatz zu diesen Beispielen und Ausführungen sehen die meisten CFOs die eigene Kompetenz in der Financial Chain als höher im Vergleich zu potenziellen Insourcern an. Sie sind somit einem Outsourcing gegenüber vielfach negativ eingestellt.

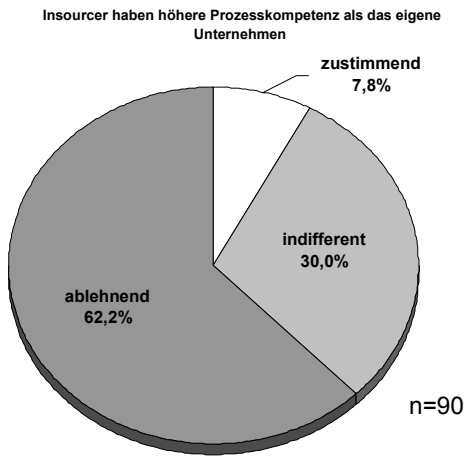

Abbildung 2: Ein Sourcing-Dienstleister beherrscht die Gestaltung und Optimierung der Teilprozesse besser als unser Unternehmen.

Um dieses verblüffende Ergebnis genauer zu verstehen, wurde betrachtet, inwieweit Outsourcing-Erfahrungen aus vergangenen Projekten einen Einfluss auf die Wahrnehmung der Kompetenz des externen Anbieters haben. Hierzu wurde zunächst der Status quo umgesetzter BPO-Projekte der Teilprozesse der Financial Chain untersucht (Abbildung 3). 


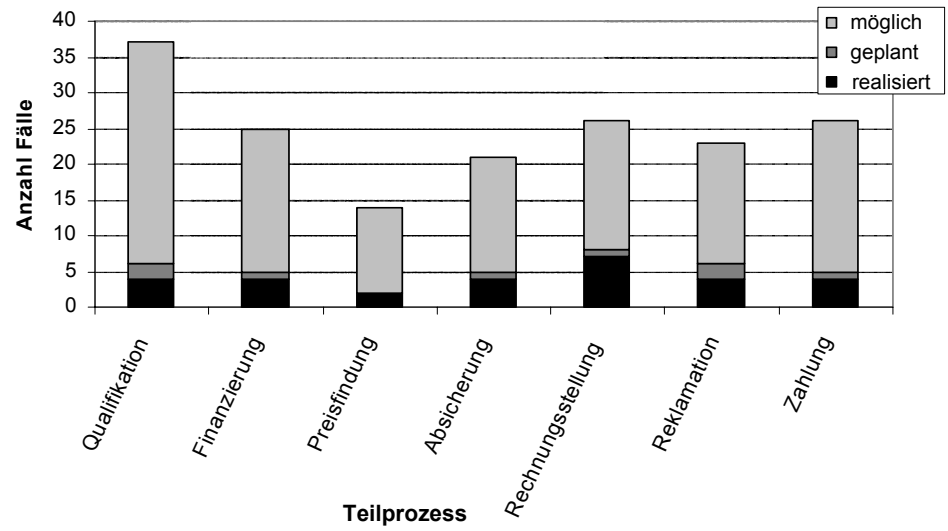

Abbildung 3: Business Process Outsourcing in der Financial Chain

Bezogen auf die grundlegende Einschätzung eines überhaupt möglichen Business Process Outsourcing wurden in der Stichprobe tatsächlich realisierte Anteile zwischen 11\% (Qualifikation) und 27\% (Rechnungsstellung) gemessen (Anzahl realisierte Projekte/Anzahl mögliche + geplante + realisierte Projekte). Die mit der Auslagerung von Teilprozessen der Financial Chain erzielten Einsparungen belaufen sich auf durchschnittlich 10,3\%. Umgesetzte Outsourcing-Projekte wurden von über der Hälfte der Unternehmen eher positiv bewertet. Betrachtet man die Differenz von erwarteten und realisierten Einsparungen, so hatten Unternehmen, die ihr Outsourcing als erfolgreich klassifizierten, im Durchschnitt Abweichungen von $0 \%$, während die ,eher erfolgreichen“ 5,6\%-Punkte, die ,indifferenten“ $8 \%$ Punkte und die ,eher gescheiterten“ 27,5\%-Punkte unter ihren Erwartungen (letztere haben Abweichungen zwischen 10\% und 40\% angegeben) lagen.

Schließlich wurde die Frage betrachtet, inwieweit Outsourcing-Erfahrung die Einschätzung der Kompetenz des Insourcers beeinflusst. Es ist ein deutlicher Unterschied im Antwortverhalten der beiden Gruppen feststellbar. So schätzen lediglich 28,5\% der CFOs mit Outsourcing-Erfahrungen die Kompetenz der InhouseAbteilung höher ein, gegenüber $60,9 \%$ der CFOs ohne Outsourcing-Erfahrung [Weit $\left.{ }^{+} 04\right]$.

Nach der Identifikation von Art und Umfang der auszulagernden Prozesse (Outsourcing von Teilprozessen, Outsourcing der gesamten Financial Chain, Beibehalten der internen Prozessbereitstellung und Bildung einer Shared Service Organization) stellt die Wahl eines geeigneten Insourcers für Teile der Financial Chain im Rahmen einer Kreuzung der Wertschöpfungsketten von Outsourcer und Insourcer eine zentrale Entscheidung dar. Als Insourcer der Financial Chain sind insbesondere Banken, andere Finanzdienstleister, IT-Dienstleister, Beratungshäuser sowie Unternehmen der gleichen Branche denkbar. 


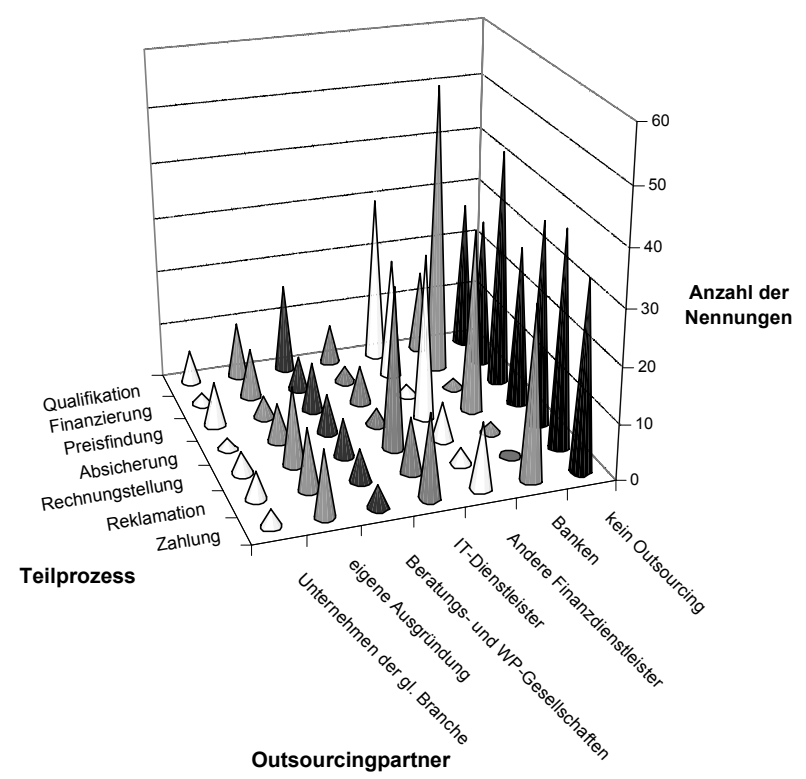

Abbildung 4: Geeignete Sourcing-Partner für die Teilprozesse der Financial Chain

Die befragten CFOs halten bei den meisten Teilprozessen "kein Outsourcing" für die beste Lösung. Für den Teilprozess „Qualifikation“ werden ,,andere Finanzdienstleister“ als geeignete Partner gesehen, für die Teilprozesse „Finanzierung“ und „Absicherung“ sind Banken als bestmögliche Partner eingestuft worden, im Fall der Finanzierung sind es sogar mehr Nennungen als für die Option ,kein Outsourcing“. IT-Dienstleister werden vor allem für den Teilprozess der „Rechnungsstellung" als viel versprechender Provider wahrgenommen. In der Zahlungsabwicklung sind - wenig überraschend - die Banken als geeignete Partner eingeschätzt worden.

\section{Ein Modell zur simultanen Bewertung der Einflussfaktoren von Sourcing-Entscheidungen im Cooperative Sourcing}

Die vorgestellte empirische Analyse zeigte, dass Economies of Scale und Scope sowie erwartete und tatsächliche Kernkompetenzen und damit zusammenhängende Lerneffekte Schlüsselfaktoren bei Outsourcing-Überlegungen darstellen. 
Was sind insbesondere gemeinsame Wirkungen der Einflussfaktoren von Sourcing auf den Sourcing-Erfolg? Wie die vorliegende Studie zeigt, bewerteten die Befragten häufig entweder Economies of Scope oder Economies of Scale bei einer Bewertung von Outsourcing-Potenzialen. Inwieweit eine simultane Betrachtung beider Einflussfaktoren zu einer besseren Sourcing-Entscheidung führen kann, ist zu untersuchen. Darauf aufbauend kann für eine Branche untersucht werden, welches geeignete Sourcing-Konstellationen sind, d. h. welche Unternehmen welche Geschäftsfunktionen kooperativ sourcen, um den Trade-off zwischen den genannten Effekten zu optimieren.

Aufbauend auf diesen Fragestellungen wird im Folgenden die Grundlage für ein formales Simulationsmodell gelegt, das Outsourcing-Entscheidungen verschiedener Akteure einer Branche oder eines Marktsegmentes nachbildet und daraus resultierende strukturelle Effekte aufzeigt. Ziel dieses Modells kann es nicht sein, detaillierte Prognosen über zukünftige Marktentwicklungen zu treffen. Vielmehr sollen mögliche Ergebnisse aufgezeigt und damit zusammenhängende Probleme frühzeitig erkannt und adressiert werden können. Gerade Akteure in der Praxis können sich über solche Modelle komplexe Zusammenhänge und mögliche Konsequenzen ihres Handelns besser vor Augen führen [Weit $\left.{ }^{+} 01\right]$.

\subsection{Modellspezifikation}

Das Modell bildet eine Menge von Unternehmen eines Marktes oder Marktsegmentes in Form von Agenten ab, die jeweils über eine individuelle Teilmenge von in diesem Marktsegment vorkommenden Geschäftsfunktionen - also Prozessteilen zur Erfüllung ihres Geschäfts - verfügen. Die Kombination verschiedener Geschäftsfunktionen innerhalb eines Unternehmens führt zu einem Geschäftsprozess, der auf die Befriedigung einer internen oder externen Nachfrage ausgerichtet ist. Die Akteure besitzen die Möglichkeit, zu bestimmten Entscheidungszeitpunkten das Outsourcing von einzelnen Teilprozessen zu evaluieren und durchzuführen. Dabei wird in einem ersten Schritt angenommen, dass die abgebildeten Unternehmen nach geeigneten Partnern zur Bildung einer Kooperation suchen, um gleichartige Teilprozesse in einem der beteiligten Unternehmen oder einer gemeinsamen Ausgründung zusammenzulegen und auf diese Weise Skaleneffekte zu erzielen.

Abbildung 5 verdeutlicht die grundlegende Struktur des Modells. Die vertikalen Säulen $i=1$ bis 5 stellen verschiedene Unternehmen dar, die je nach Branche über ein mehr oder weniger ähnliches Set von Geschäftsfunktionen (symbolisiert durch die vertikale Achse ( $k=1$ bis 7)) verfügen. So können sich Autohäuser hinsichtlich der Financial Chain bspw. darin unterscheiden, ob sie eine Absatzfinanzierung im Funktionsportfolio haben oder nicht. Im Zuge eines Cooperative Sourcing können sich die Unternehmen nun dazu entschließen, letztlich parallel vorgehaltene, hinreichend identische Geschäftsfunktionen in einer Kooperation $\left(M_{k m}\right)$ zu sourcen 
und somit Einsparungen durch die Vermeidung redundanter Geschäftsfunktionen und die Realisierung von Skaleneffekten zu erzielen (beispielhaft sind in Abbildung 5 drei verschiedene Cooperative-Sourcing-Kombinationen dargestellt, die Menge $M_{5 l}$ ist die erste $(m)$ und hier einzige auf dem Markt etablierte Kooperation für Geschäftsfunktion $k=5$ (z. B. die Rechnungsstellung) und umfasst die beteiligten Akteure 3, 4 und 5).

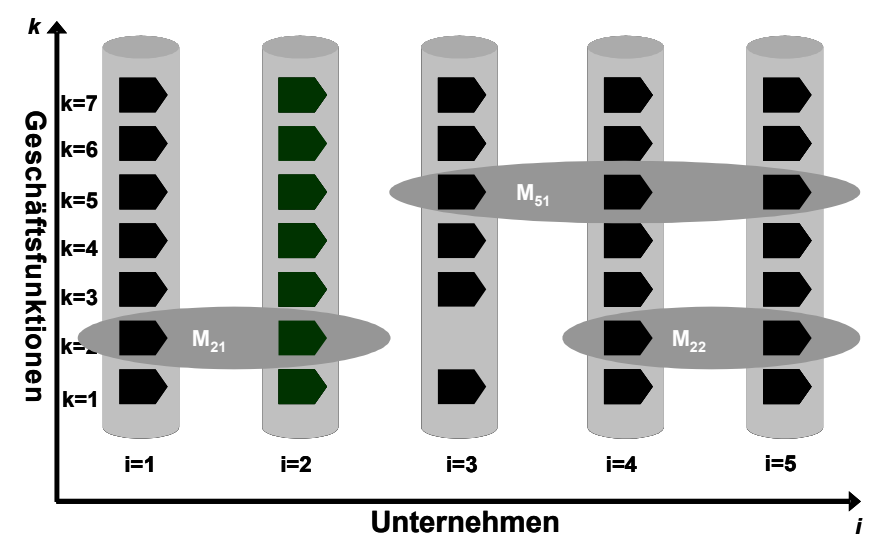

Abbildung 5: Cooperative Sourcing von Geschäftsprozessteilen

\begin{tabular}{|l|l|l|}
\hline$i, j, I,|I|$ & $\begin{array}{l}\text { Indizes, Menge und Anzahl abge- } \\
\text { bildeter Akteure }\end{array}$ & $\begin{array}{l}i, j \in I \\
i, j=1 \ldots|I|\end{array}$ \\
\hline$k, l, K,|K|$ & $\begin{array}{l}\text { Indizes, Menge und Anzahl der im } \\
\text { abgebildeten Markt verfügbaren } \\
\text { Geschäftsfunktionen }\end{array}$ & $\begin{array}{l}k, l \in K \\
k, l=1 \ldots|K|\end{array}$ \\
\hline$p_{i k} \in\{0 ; 1\}$ & $\begin{array}{l}\text { Indikator (Binärvariable), ob Ak- } \\
\text { teur } i \text { Geschäftsfunktion } k \text { imple- } \\
\text { mentiert hat }\end{array}$ & $\left.\begin{array}{l}\text { Funktionsportfolio von } i: \\
\vec{p}_{i}=\left\{p_{i l}, \ldots, p_{i k}, \ldots, p_{i|K|}\right.\end{array}\right\}$ \\
\hline
\end{tabular}

Tabelle 3: Akteure und Geschäftsfunktionen

Um die in der Literatur und der vorgestellten Empirie maßgeblichen OutsourcingFaktoren (Treiber und Inhibitoren) abbilden zu können, werden die einzelnen Teilprozesse mit Produktionskostenfunktionen ausgestattet, die über einen fixen und einen variablen Anteil verfügen. Aus Komplexitätsgründen wird zunächst vereinfachend eine lineare Kostenfunktion angenommen. Beim Cooperative Sourcing gleichartiger Prozesse kommt dann jeweils die Technologie desjenigen Partners zum Tragen, welche die niedrigsten Gesamtproduktionskosten für das gemeinsame Prozessvolumen bietet. 


\begin{tabular}{|c|c|c|}
\hline$C_{i k}^{P}$ & $\begin{array}{l}\text { Produktionskos- } \\
\text { ten von Funkti- } \\
\text { on } k \text { in Unter- } \\
\text { nehmen } i\end{array}$ & $C_{i k}^{P}=K_{i k}^{F}+c_{i k}^{P} \cdot x_{i k}$ \\
\hline$K_{i k}^{F}, c_{i k}^{P}$ & \multicolumn{2}{|c|}{ Fixkosten und variable Stückkosten von Funktion $k$ des Akteurs $i$} \\
\hline$x_{i k}$ & \multicolumn{2}{|c|}{$\begin{array}{l}\text { Output von Prozess } k \text { in Unternehmen } i \text {, zunächst als intertempo- } \\
\text { ral konstant angenommen }\end{array}$} \\
\hline$C_{k m t}^{P M}$ & $\begin{array}{l}\text { Produktionskos- } \\
\text { ten der Koope- } \\
\text { ration } k m \text { in Pe- } \\
\text { riode } t\end{array}$ & $C_{k m t}^{P M}:=\min _{i \in M_{k m t}} C_{i k}^{P}\left(\sum_{i \in M_{k m t}} x_{i k}\right)$ \\
\hline$M_{k m t}$ & \multicolumn{2}{|c|}{$\begin{array}{l}\text { Menge der an der Kooperation } \mathrm{km} \text { in Periode } t \text { beteiligten Unter- } \\
\text { nehmen }\end{array}$} \\
\hline
\end{tabular}

Tabelle 4: Produktionskosten

Es wirken nun verschiedene, teilweise gegenläufige Einflussfaktoren auf die Vorteilhaftigkeit der Sourcing-Entscheidung. Einerseits können durch die Bündelung der Geschäftsfunktionen Economies of Scale durch eine stärkere Inanspruchnahme der einzelnen Funktion realisiert werden. Andererseits sind Transaktionskosten in Form von einmaligen Verhandlungs- und Anpassungskosten in der Kontraktphase sowie laufende Schnittstellenkosten und Koordinationsaufwendungen durch die Abstimmung mit den Sourcing-Partnern zu tragen.

\begin{tabular}{|c|c|}
\hline$t=t^{*}$ (Kontraktphase) & $t>t^{*}$ (Kooperationsphase) \\
\hline Verhandlungskosten & Koordinationskosten \\
\hline Anpassungskosten & Schnittstellenkosten \\
\hline
\end{tabular}

Tabelle 5: Anfallende Transaktionskosten bei Cooperative Sourcing

Erstverhandlungs- und laufende Koordinationskosten werden in Abhängigkeit der Kooperationsgröße (Zahl der Teilnehmer) [Beim $\left.{ }^{+} 04\right]$ und der Erfahrung des jeweiligen Unternehmens mit vergangenen Outsourcing-Projekten (Lerneffekte, degressiv abnehmend, [EwWa03; Levi88]) formuliert. 


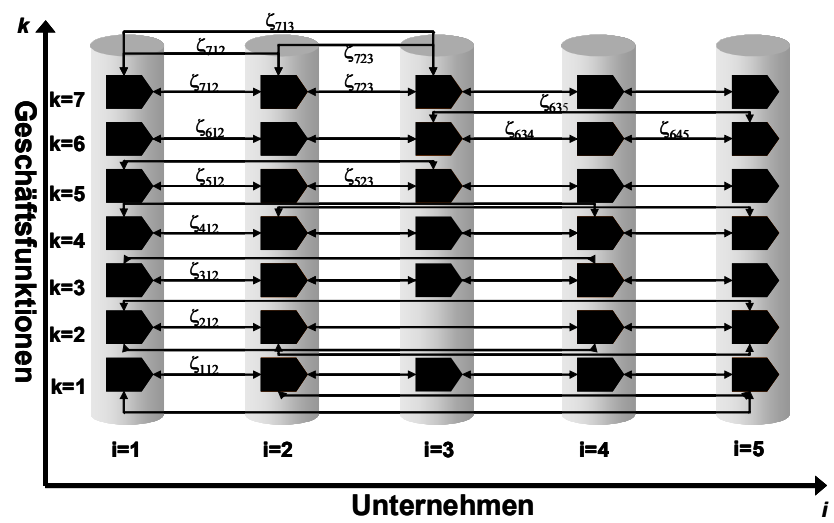

Abbildung 6: Standardisierungsbedarf zwischen Teilprozessen (nicht vollständig dargestellt)

Die einmaligen Anpassungskosten, welche durch eine Standardisierungsbedarfskennziffer $\zeta_{k i j t}$ determiniert werden, beschreiben Aufwendungen (,organisationale Extegrationskosten“), die ein Unternehmen $i$ zu tragen hat, wenn es zum Zeitpunkt $t$ einen Teilprozess $k$ auslagert und sich auf den vorgegebenen Standardprozess eines anderen Unternehmens einlassen muss (Abbildung 6). Ohne diese Prozessstandardisierung wäre das Erzielen von Skaleneffekten nur in unzureichendem Maße möglich.

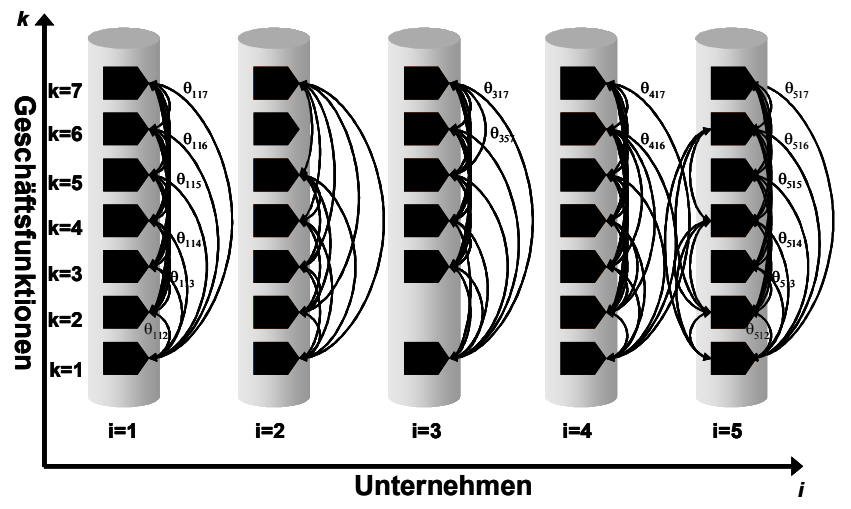

Abbildung 7: Modularitätsgrad zwischen Teilprozessen (nicht vollständig dargestellt)

Die Schnittstellenkosten zwischen einzelnen Geschäftsfunktionen (operationalisiert durch den Modularitätsgrad $\theta_{i k l}$ ) resultieren aus Aufgabeninterdependenzen aufgrund erforderlicher Interaktionen zwischen outgesourcten und ingesourcten Teilprozessen, um einen aus mehreren Geschäftsfunktionen bestehenden Ge- 
schäftsprozess zu formen (Economies of Scope). So können beispielsweise zusätzliche Kosten durch die Absicherung von Datenflüssen zwischen von verschiedenen Unternehmen bereitgestellten Geschäftsfunktionen entstehen oder Effizienzverluste durch das Aufbrechen eines Ressourcenverbundes eintreten (Abbildung 7).

\begin{tabular}{|c|c|c|c|}
\hline$C_{i k m t}^{A D}$ & $\begin{array}{l}\text { Anpassungs- } \\
\text { kosten }\end{array}$ & $\begin{array}{l}C_{i k m t}^{A D}=\zeta_{k i *^{*} t} \cdot \\
\text { mit } j^{*}=\text { Insour } \\
\text { de } t\end{array}$ & der Kooperation $k m$ in Perio- \\
\hline$c_{k}^{A D}$ & $\begin{array}{l}\text { Standardisierter } \\
\text { Anpassungs- } \\
\text { kostenfaktor für } \\
\text { Funktionstyp } k\end{array}$ & $\zeta_{k i j t}=[0,0 ; 1,0]$ & $\begin{array}{l}\text { Standardisierungsbedarf zwi- } \\
\text { schen den Geschäftsfunktio- } \\
\text { nen } k \text { der Akteure } i \text { und } j \text { (vor } \\
\text { Anpassung) in Periode } t\end{array}$ \\
\hline$C_{i t}^{I F}$ & \begin{tabular}{l|l} 
Schnitt- & \\
stellen- & $C$ \\
kosten von & \\
Akteur $i$ & \\
\end{tabular} & \multicolumn{2}{|c|}{$C_{i t}^{I F}=\frac{1}{2} \sum_{m=0}^{|M|} \sum_{k \in K} \sum_{l \in K} \theta_{i k l} \cdot c^{I F} \cdot \operatorname{sign}\left(z_{i k m t}-z_{i l m t}\right) \forall i, t$} \\
\hline$c^{I F}$ & \multicolumn{3}{|c|}{ Standardisierter Schnittstellenkostenfaktor } \\
\hline $\begin{array}{l}\theta_{i k l}= \\
{[0,0 ; 1,0[}\end{array}$ & \multicolumn{3}{|c|}{$\begin{array}{l}\text { Interdependenz zwischen Geschäftsfunktionen } k \text { und } l \text { in Unter- } \\
\text { nehmen } i\end{array}$} \\
\hline$z_{i k m t}$ & \multicolumn{3}{|c|}{$\begin{array}{l}\text { Binärvariable, die angibt, ob Prozess } k \text { von Akteur } i \text { in Periode } t \\
\text { durch Kooperation } \mathrm{km} \text { ausgeführt wird. }\end{array}$} \\
\hline$C_{i k m t}^{V}$ & $\begin{array}{l}\text { Verhandlungs- } \\
\text { kosten für Ak- } \\
\text { teur } i \text { in Koope- } \\
\text { ration } k m \text { in Pe- } \\
\text { riode } t\end{array}$ & \multicolumn{2}{|c|}{$C_{i k m t}^{V}=c_{k}^{V} \cdot\left|M_{k m}\right|^{\alpha} \cdot o_{i t}^{\beta}$ mit $\alpha>1$ und $\beta<0$} \\
\hline$o_{i t}$ & \multicolumn{3}{|c|}{ Anzahl von Outsourcing-Projekten von Akteur $i$ bis Periode $t-1$} \\
\hline$c_{k}^{V}$ & \multicolumn{3}{|c|}{ Standardisierter Verhandlungskostenfaktor } \\
\hline$C_{i k m t}^{C}$ & $\begin{array}{l}\text { Koordinations- } \\
\text { kosten }\end{array}$ & \multicolumn{2}{|c|}{$C_{i k m t}^{C}=\gamma \cdot C_{i k m t}^{V}$ mit $0<\gamma<1$} \\
\hline
\end{tabular}

Tabelle 6: Spezifikation verschiedener Arten von Transaktionskosten

Nach der Modellierung der Akteure, Teilprozesse und Parameter zur Bestimmung der entscheidungsrelevanten Kostenfaktoren können intertemporale Entscheidungsfunktionen entwickelt werden, die den Unternehmen als Grundlage für Cooperative-Sourcing-Verhandlungen dienen. Formel 1 gibt ein statisches geschäftsfunktionsorientiertes Entscheidungskalkül $(C I R=$ Kalkulationszinssatz) unter der 
Annahme an, dass die gewählte Kooperation über die Vertragslaufzeit $T^{\text {CoSo }}$ unverändert bleibt.

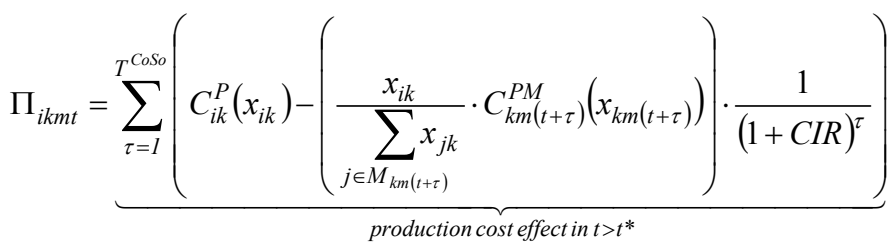

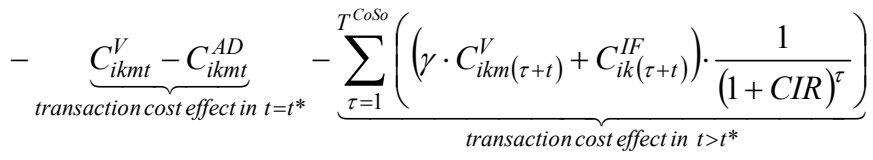

Den bei Cooperative Sourcing gegenüber der Selbsterstellung der Geschäftsfunktion durch Economies of Scale entstehenden Einsparungen stehen die beschriebenen einmaligen und laufenden Transaktionskosten, welche aus Economies of Scope und dem Verhandlungsaufwand resultieren, gegenüber.

\subsection{Ein Szenario zur Anwendung des Modells}

Innerhalb des Modells wirkt eine Reihe von unterschiedlichen Faktoren, deren Beziehung zueinander im Folgenden kurz dargestellt und anhand des empirischen Beispieles der Financial Chain diskutiert werden soll.

Die zu realisierenden Einsparungen können als Economies of Scale durch die $\mathrm{Zu}$ sammenlegung von Geschäftsfunktionen erreicht werden. Je nach individueller Ausgestaltung der Produktionskostenfunktionen der beteiligten Partner werden hier Einsparungen erzielt. So ist in der Financial Chain beispielsweise durch die Bündelung der Rechnungsstellung eine Kostenreduktion durch die gemeinsame Nutzung von Druck- und Versandkapazitäten möglich. Innerhalb des Modells wird angenommen, dass jeweils die kostengünstigste Produktionstechnik verwendet wird. So ist für die Rechnungsstellung denkbar, dass der Partner mit einer ohnehin bereits höheren Anzahl an Rechnungen und einem größeren Versandzentrum diese Funktion für ein oder mehrere Partner mit übernimmt.

Auf der anderen Seite sind jedoch Kosten zu berücksichtigen, welche die Vorteilhaftigkeit des Cooperative Sourcing beeinflussen. So ist zunächst ein geeigneter Partner zu finden, mit welchem im Rahmen der Kontraktphase einerseits Verhandlungen über die spezifische Ausgestaltung der gemeinsamen Geschäftsfunktion und andererseits über die Verteilung der Einsparungen zu führen sind. Weiterhin ist es notwendig, die bestehenden Geschäftsfunktionen gegebenenfalls aneinander anzupassen. Dabei muss sich in der Regel das auslagernde Unternehmen an den „Standardprozess“ des Partners anpassen. Diese einmaligen Kosten fallen im Rahmen der Kontraktphase an. 
Laufende Kosten fallen in der Sourcing-Beziehung durch Koordinations- und Schnittstellenkosten an. Änderungen an der grundlegenden Gestaltung der Geschäftsfunktion beispielsweise durch ein neues Datenfeld auf der Rechnung sind nur in Abstimmung mit dem oder den Partnern zu realisieren und bringen Koordinationsaufwand mit sich.

Weiterhin sind gegebenenfalls laufende Schnittstellenkosten zu tragen. So ist die Rechnungsstellung beispielsweise eng mit dem Prozess der Preisfindung verknüpft, in dem die in der Rechnung zu stellenden Preise festgesetzt werden. Eine enge Abstimmung beider Prozesse und damit die Vermeidung fehlerhaft gestellter Rechnungen ist vorteilhaft und resultiert aus Verbundvorteilen (Economies of Scope). Da innerhalb der Sourcing-Beziehung diese Abstimmung im Gegensatz zur internen Abstimmung über Unternehmensgrenzen hinweg geschehen muss, fallen hier im Vergleich gegebenenfalls höhere Übertragungskosten an, da im genannten Beispiel etwa die Datenübertragung zwischen den Unternehmensnetzwerken und den Geschäftsfunktionen Preisfindung und Rechnungsstellung zusätzlich abgesichert werden muss.

Insgesamt wird innerhalb des vorgestellten Modells ein kooperatives Sourcing genau dann vorteilhaft sein, wenn die zusammenzulegenden Geschäftsfunktionen bereits hoch standardisiert sind, umfangreiche Economies of Scale realisierbar sowie Verbundeffekte zwischen in- und outgesourcten Funktionen vergleichsweise gering sind. Dabei werden die Vorteile mit zunehmender Größe der Kooperation abnehmen, da der Produktionskostenvorteil generell durch zusätzliche Teilnehmer degressiv abnimmt, die Koordinationskosten dagegen steigen.

\subsection{Ausblick}

Das vorliegende Modell ist in einem nächsten Schritt als Simulationsmodell implementierbar, welches autonome Entscheidungen und Verhandlungen der einzelnen Unternehmen abbildet und die resultierenden Gleichgewichte aufzeigt. Dazu ist die Entwicklung von agentenbasierten Entscheidungskalkülen notwendig, welche die zukünftigen Nettoeffekte einer Outsourcing-Entscheidung zu antizipieren helfen. Im beschriebenen Modell (Gleichung (1)) wird eine eingegangene Kooperation zunächst als statisch angenommen, d. h. es gibt keine Erwartungen über zukünftige Variationen der Partnerstruktur; eine entsprechende Erwartungsbildung über mögliche Veränderungen (Externalitäten, Volatilität des Geschäftsumfeldes) im Laufe der Beziehung wird in einem späteren Schritt integriert. Weiterhin muss in der Entscheidungsfunktion berücksichtigt werden, dass die potenziellen Partner in einem wettbewerblichen Verhältnis untereinander stehen („Coopetition“). Zu den Transaktionskostenbestandteilen kommt dann ein weiterer Faktor, die Agency-Kosten, hinzu, die davon abhängen, wie stark die Partner im Wettbewerb stehen und wie hoch der potenzielle Schaden wäre, wenn ein Wettbewerber die gemeinsame Prozessabwicklung opportunistisch ausnutzen würde. 
Aufgrund der streng formalen Entwicklung ist parallel dazu die Aufstellung eines mathematischen Optimierungsansatzes möglich, der bei gegebenen Parameterkonstellationen die optimale Sourcing-Konfiguration für das betrachtete Marktsegment und die Teilprozesse bietet (für eine Unternehmung in einem einfacheren Modell dargestellt in [Knol93]). Aus einem Vergleich des Optimierungs- und des Simulationsergebnisses lassen sich strukturell diejenigen Situationen erkennen, in denen autonom entscheidende Akteure (bspw. aufgrund von Externalitäten) kein optimales System erreichen [Weit04].

In Zukunft wird die Beschränkung auf lineare Kostenfunktion aufgehoben und für die jeweilige Geschäftsfunktion besser geeignete Funktionen implementiert werden. In erweiterten Modellvarianten können auch existierende Business Service Provider als potenzielle Insourcer auftreten, es werden Wettbewerbseffekte zwischen Sourcing-Partnern berücksichtigt oder die Akteure können ihre zusammengelegten Services auch marktfremden Unternehmen in Form des Value Chain Crossing anbieten.

\section{Zusammenfassung}

Ein Outsourcing von Teilprozessen der Financial Chain zu spezialisierten Anbietern mit potenziell höherer Kompetenz ist bislang nur selten erfolgt. Firmen, die bereits Teilprozesse outgesourct haben, konnten jedoch teilweise umfangreiche operative Kosteneinsparungen realisieren. Je nach betrachtetem Teilprozess werden Banken und IT-Dienstleister als geeignete Insourcer gesehen. Obwohl das Management der Financial Chain nicht zu den Kernprozessen der Unternehmen zu zählen ist, tendieren viele CFOs dazu, die eigene Prozesskompetenz im Vergleich zu externen Experten systematisch zu überschätzen. Dies kann als interne kulturelle Barriere betrachtet werden, die ein Outsourcing von Teilprozessen verhindert. Es muss erkannt und akzeptiert werden, dass Spezialisierung auch bedeutet, dass andere Unternehmen manche Prozesse besser abwickeln als das eigene Unternehmen.

Kooperatives Sourcing kann den nächsten Schritt zu einer grundlegenden Neustrukturierung der Wertschöpfungskette darstellen. Falls Economies of Scale angestrebt werden, ist es möglich, dass ein Unternehmen beispielsweise davon profitiert, wenn ein Mitbewerber mit ähnlichen Prozessen auf der gleichen Plattform abgewickelt wird, um ein höheres Volumen zu generieren. Um allerdings solche Netzwerke breit zu etablieren, sind zunächst Sourcing-Modelle zu entwickeln, die eine simultane Berücksichtigung der Einflussfaktoren zulassen. Ein erster Ansatz für ein entsprechendes Modell wurde im Rahmen dieses Beitrags vorgestellt.

\section{Danksagung}

Die Autoren danken den Partnern des E-Finance Lab für ihre Unterstützung. 


\section{Literatur}

[Baru ${ }^{+}$95] Barua, A.; Kriebel, C. H.; Mukhopadhyay, T.: Information Technologies and Business Value: An Analysis and Empirical Investigation. In: Information Systems Research, 6, 1, 1995, S. 3-23.

[Beim ${ }^{+}$04] Beimborn, D.; Fladung, R.; König, W.: An optimization framework for efficient information supply in the academic sector, 8th Pacific Asia Conference on Information Systems (PACIS), Shanghai, China, 2004.

[BrRo03] Brown, C. W.; Ross, J. W.: Designing a Process-Based IT Organization. In: Information Strategy: The Executive's Journal, 2003.

[Bruc98] Bruch, H.: Outsourcing - Konzepte und Strategien, Chancen und Risiken. Gabler, Wiesbaden, 1998.

[CaHa02] Cachon, G.; Harker, P.: Competition and Outsourcing with Scale Economies. In: Management Science, 48, 10, 2002, S. 1314-1333.

[CuWe03] Currie, W. L.; Weerakkody, V.: Guest Editorial. In: Business Process Management Journal, 9, 6, 2003.

[DeCu03] Desai, B.; Currie, W. L.: Application Service Providers: A model in Evolution, 5th International Conference on Electronic Commerce, Pittsburgh, 2003.

[DiGo ${ }^{+}$04] Dibbern, J.; Goles, T.; R., H.; Jayatilaka, B.: Information Systems Outsourcing: A Survey and Analysis of the Literature. In: The DATA BASE for Advances in Information Systems, 35, 4, 2004, S. 6-102.

[DiHe01] Dibbern, J.; Heinzl, A.: Outsourcing der Informationsverarbeitung im Mittelstand: Test eines multitheoretischen Kausalmodells. In: Wirtschaftsinformatik, 43, 4, 2001, S. 339-350.

[Ear196] Earl, M. J.: The Risks of Outsourcing IT. In: Sloan Management Review, 37, 3, 1996, S. 26-32.

[EwWa03] Ewert, R.; Wagenhofer, A.: Interne Unternehmensrechnung. Springer, Berlin, Heidelberg u. a., 2003.

[FeWi98] Feeny, D. F.; Willcocks, L. P.: Re-designing the IS Function around Core Capabilities. In: Long Range Planning, 31, 3, 1998, S. 354-367.

[HaMe00] Halvey, J. K.; Melby, B. M.: Business Process Outsourcing - Process, Strategies and Contracts. John Wiley \& Sons, New York, 2000.

[Hein02] Heinrich, L. J.: Informationsmanagement. R. Oldenbourg Verlag, München, 2002.

[KeKr $\left.{ }^{+} 02\right]$ Kern, T.; Kreijger, J.; Willcocks, L. P.: Exploring ASP as sourcing strategy: theoretical perspectives, propositions for practice. In: Journal of Strategic Information Systems, 11, 2002, S. 153-177. 
[KeWi96] Kern, T.; Willcocks, L. P.: The Enabling and Determining Environment: Neglected Issues in an IT/IS Outsourcing Strategy, European Conference on Information Systems (ECIS), Lisbon, Portugal, 1996.

[Kno193] Knolmeyer, G.: Modelle zur Unterstützung von Outsourcing-Entscheidungen. In: Kurbel, K. (Ed.): Wirtschaftsinformatik'93. Springer, Heidelberg 1993, S. 70-83.

[LaHi93] Lacity, M. C.; Hirschheim, R.: Information Systems Outsourcing - Myths, Metaphors and Realities. John Wiley \& Sons Ltd., Chichester, 1993.

[Laci+96] Lacity, M. C.; Willcocks, L. P.; Feeny, D. F.: The Value of Selective IT Sourcing. In: Sloan Management Review, 37, 3, 1996, S. 13-25.

[LeHu+03] Lee, J.-N.; Huynh, M. Q.; Kwok, R. C.-W.; Pi, S.-M.: IT Outsourcing Evolution - Past, Present, and Future. In: Communications of the ACM, 44, 5, 2003, S. 84-85.

[Levi88] Levinthal, D.: A Survey of Agency Models of Organization. In: Journal of Economic Behavior and Organization, 9, 2, 1988, S. 153-186.

[LoVe95] Loh, L.; Venkatraman, N.: An empirical study of information technology outsourcing: Benefits, risk and performance implications, Sixteenth International Conference on Information Systems, Amsterdam, 1995, S. 277-288.

[MaMe02] Matiaske, W.; Mellewigt, T.: Motive, Erfolge und Risiken des Outsourcings Befunde und Defizite der empirischen Outsourcing-Forschung. In: Zeitschrift für Betriebswirtschaft (ZfB), 72, 6, 2002, S. 641-659.

[Melv+04] Melville, N.; Kraemer, K. L.; Gurbaxi, V.: Review: Information Technology and Organizational Performance: An Integrative Model of IT Business Value. In: MIS Quarterly, 28, 2, 2004, S. 283-322.

[PaWi81] Panzar, J.; Willig, R.: Economies of Scope. In: American Economic Review, 71, 2, 1981, S. 268-272.

[Pfaf ${ }^{\dagger}$ 03] Pfaff, D.; Skiera, B.; Weitzel, T.: Datenintegration in Finanzprozessen, Informatik 2003 - Innovative Informatikanwendungen, Frankfurt, 2003.

[Port85] Porter, M. E.: Competitive Advantage. Free Press, New York, 1985.

[PrHa90] Prahalad, C. K.; Hamel, G.: The Core Competence of the Corporation. In: Harvard Business Review, May-June 1990, 1990.

[Ried03] Riedl, R.: Begriffliche Grundlagen des Business Process Outsourcing. In: Information Management \& Consulting, 18, 3, 2003, S. 6-10.

[Rock ${ }^{+}$96] Rockart, J. F.; Earl, M. J.; Ross, J. W.: Eight Imperatives for the IT Organization. In: Sloan Management Review, 38, 1, 1996.

[RoCo03] Rouse, A.; Corbitt, B.: Minimising risks in IT outsourcing: Choosing target services, 7th Pacific Asia Conference on Information Systems (PACIS), Adelaide, South Australia, 2003.

[RoCo04] Rouse, A.; Corbitt, B.: IT-supported business process outsourcing (BPO): The good, the bad and the ugly, The 8th Pacific Asia Conference on Information Systems (PACIS), Shanghai, 2004. 
[Scho97] Schott, E.: Markt und Geschäftsbeziehung beim Outsourcing - Eine marketingorientierte Analyse für die Informationsverarbeitung. Deutscher Universitätsverlag, Wiesbaden, 1997.

[Skie ${ }^{+}$04] Skiera, B.; König, W.; Gensler, S.; Weitzel, T.; Beimborn, D.; Blumenberg, S.; Franke, J.; Pfaff, D.: Financial Chain Management - Prozessanalyse, Effizienzpotenziale und Outsourcing. Books on Demand, Norderstedt, 2004.

[Tebb03] Tebboune, D. E. S.: Application service provision: origins and development. In: Business Process Management Journal, 9, 6, 2003, S. 722-734.

[Teec80] Teece, D.: Economies of scope and the scope of the enterprise. In: Journal of Economic Behavior and Organization, 1, 1980, S. 223-247.

[Weer ${ }^{+} 03$ ] Weerakkody, V.; Currie, W. L.; Ekanayake, Y.: Re-engineering business processes through Outsourcing: The Knowledge Potential. In: Information Systems Management, (Summer), 2003, S. 7-15.

[Weit04] Weitzel, T.: The economics of standards in information networks. Springer Physica, Heidelberg, Berlin, New York, 2004.

[Weit ${ }^{+}$04] Weitzel, T.; Beimborn, D.; Franke, J.: Outsourcing the Financial Chain: an Empirical Analysis of Sourcing and Partnering Potentials, Tenth Americas Conference on Information Systems, New York, 2004.

[Weit $\left.{ }^{+} 01\right]$ Weitzel, T.; Son, S.; König, W.: Infrastrukturentscheidungen in vernetzten Unternehmen: Eine Wirtschaftlichkeitsanalyse am Beispiel von X.500 Directory Services. In: Wirtschaftsinformatik, 43, 4, 2001, S. 371-382.

[Will ${ }^{+}$04] Willcocks, L. P.; Hindle, J.; Feeny, D.; Lacity, M. C.: IT and Business Process Outsourcing: The Knowledge Potential. In: Information Systems Management, 21, 3, 2004, S. 7-15. 
